\title{
Viewpoint \\ A fly's eye view of tumor progression and metastasis
}

Karen L Schmeichel

Lawrence Berkeley National Laboratory, Berkeley, CA 94720,USA

Corresponding author: Karen L Schmeichel (email: KLSchmeichel@lbl.gov)

Published: 27 January 2004

Breast Cancer Res 2004, 6:82-83 (DOI 10.1186/bcr763)

(C) 2004 BioMed Central Ltd (Print ISSN 1465-5411; Online ISSN 1465-542X)

\section{Introduction}

When developing appropriate models to study tumor progression and metastasis it is essential to balance concerns of tissue fidelity and physiological relevance with the experimental accessibility of the system. Existing models are currently being re-evaluated given an increasing awareness of tumor complexity: in addition to the tumor cells themselves, their neighboring cells and the surrounding stroma are now recognized as important regulators of cancer progression [1]. With these added complexities in mind, two complementary publications have recently made a compelling case for the use of Drosophila melanogaster in understanding tumor progression and the metastatic process $[2,3]$.

Previous studies using Drosophila to model cancer revealed a collection of individual genes [e.g., discs large $(d / g)$, lethal giant larvae (/gl), scribble (scrib), Ras and lats] that when homozygous mutant, resulted in tumor-like overproliferation in epithelial cell-rich larval imaginal discs (reviewed in $[4,5]$ ). The papers in question both suggest that instead of analyzing the effects of cancer-promoting mutations in the context of an entirely mutant animal or tissue, it would be more informative to generate mosaic animals in which cancer-disposing mutations are introduced, either alone or in specific combinations, in only a subset of cells. Such a model would not only shed light on the multi-step nature of tumor progression but would also offer a reasonable approximation of the clonal nature of human cancers.

\section{Modeling tumor progression and metastasis in Drosophila melanogaster}

While the oncogenic properties of activated Ras are widely appreciated (with normal cellular Ras playing a role in proliferation, differentiation, and cell survival), the function of the epithelial cell polarity gene scrib as a tumor suppressor is only starting to be understood. Using a
MARCM (mosaic analysis with a repressible cell marker) [6], both the laboratories generated flies in which activated Ras ( $\operatorname{Ras}^{A C T}$ ) and mutant scrib genes were expressed (either alone or together) in a subset of cells specifically in the developing eye imaginal discs. These animals were designed such that only those cells displaying the engineered mutation(s) would express a green fluorescent protein reporter gene, thereby facilitating visual tracking of affected tissue.

Using this approach, both studies show that, analogous to previous results in the homozygous mutant Ras tissues, Ras ${ }^{A C T}$ cell clones in mosaic animals display a notable overproliferation in the eye imaginal disk of late stage larvae. The scrib- mosaic clones, however, deviate significantly from previous studies in homozygous animals: instead of displaying grossly neoplastic overproliferation, the mutant cells form small disordered foci that never extend beyond original clonal region of expression. Brumby and Richardson [3] elaborate upon this observation to show that overproliferation of scrib- cells is suppressed in these mosaic tissues by activation of JNK-mediated apoptotic pathways in a fashion that is not cell autonomous but rather depends upon the presence of surrounding wild-type cells.

When $\mathrm{Ras}^{\mathrm{ACT}}$ and scrib- mutations were introduced simultaneously in the same cells, both laboratories observed a dramatic synergistic effect in which doubly mutant cells experience overgrowth drastically in excess of clones displaying either mutation alone. Affected cells grew such that the eye imaginal discs expanded to many times their normal size, eventually fusing with brain lobes and other imaginal tissues and resulting in pupal lethality. Upon further examination, Pagliarini and colleagues [2], showed that in addition to this hyperproliferative effect, doublymutant cells are capable of system-wide spread and metastasis to secondary sites in the animal (including the ventral nerve cord, the leg discs, the tracheal vasculature 
and the hemolymph). Other purely growth-promoting mutations, in either E2F/DP or Cyclin E, could not substitute for RasACT in scrib- cells nor could insults to MAPK pathway effectors, such as PI3 kinase, Ral, Rho, Rac or Cdc 42, however, activated Notch exerted a Ras ${ }^{A C T}$-like hyperplastic effect in scrib- cells [3]. On the other hand, when mutations in other genes known to function in the scrib pathway (i.e., dlg, lgl, bazooka and stardust) are expressed in conjunction with Ras ${ }^{\mathrm{ACT}}$, the affected tissue still exhibits hyperproliferative and metastatic defects.

\section{Conclusions and future directions}

At the very least, the studies described by these groups provide an elegant proof-of-principle for the multi-step theory of cancer development in a model that also takes into account tissue context and the clonal nature of tumor progression. These papers clearly elucidate a combination of mutations in Ras and scribble pathways that are, in and of themselves, sufficient to cause tumor progression and metastasis in flies. In doing so, they provide further proof that maintenance of intrinsic cellular polarity can effectively counteract the devastating proliferative defects associated with mutations in global signaling molecules such as Ras. Moreover, since the effects of Ras ${ }^{\mathrm{ACT}}$ were not mimicked by known downstream Ras targets, these studies may have revealed a novel cellular mechanism by which Ras cooperates with scrib. Given the suppressive effects of wild-type cells on scrib mutant clones, these studies serve as a striking illustration of the importance of tissue context in tumor progression. Understanding not only how mutations affect tumor cells themselves, but also how surrounding wild-type cells interact with, and possibly protect, the tumor cells is of crucial importance in understanding the complexities of cancer. In the future it will be possible to generate specific and systematic pairings (triplings, etc.) of candidate oncogenes and tumor suppressors, and to evaluate their combinatorial effects on proliferation, apoptosis, tissue architecture and metastasis in a physiologically relevant context. Additional Drosophila-based screens for other genes that compliment Ras and scrib pathways during tumor progression are likely already underway.

Is Drosophila the best model for study of tumor progression and metastasis? Obviously, as with any model, this system has its limitations. For example, fundamental differences between the hemolymph system in flies and the mammalian vasculature and circulatory system bring into question the utility of this organism to accurately model metastatic processes. However, given the ease of analysis and the genetic capabilities presented in these two papers, Drosophila is even more relevant to cancer research than ever.

\section{Competing interests}

None declared.

\section{References}

1. Bissell MJ, Radisky D: Putting tumours in context. Nat Rev Cancer 2001, 1:46-54.

2. Pagliarini RA, $\mathrm{Xu}$ T: A genetic screen in Drosophila for metastatic behavior. Science 2003, 302:1227-1231.

3. Brumby AM, Richardson HE: scribble Mutants cooperate with oncogenic Ras or Notch to cause neoplastic overgrowth in Drosophila. Embo J 2003, 22:5769-5779.

4. Humbert P, Russell S, Richardson H: Dlg, Scribble and Lgl in cell polarity, cell proliferation and cancer. Bioessays 2003, 25: 542-553.

5. Potter CJ, Turenchalk GS, Xu T: Drosophila in cancer research An expanding role. Trends Genet 2000, 16:33-39.

6. Lee T, Luo L: Mosaic analysis with a repressible cell marker for studies of gene function and neuronal morphogenesis. Neuron 1999, 22:451-461.

\section{Correspondence}

Karen Schmeichel, Lawrence Berkeley National Laboratory, Berkeley, CA 94720,USA. Tel: +1 510486 4368; fax: +1 510486 5586; e-mail: KLSchmeichel@lbl.gov 